\title{
Triterpenoid saponin flaccidoside II from Anemone flaccida triggers apoptosis of NFI-associated malignant peripheral nerve sheath tumors via the MAPK-HO-I pathway
}

\author{
This article was published in the following Dove Press journal: \\ OncoTargets and Therapy \\ 5 April 2016 \\ Number of times this article has been viewed
}

\author{
Lin-tao Han' \\ Yin Fang' \\ Yan $\mathrm{Cao}^{2}$ \\ Feng-hua $\mathrm{Wu}^{\prime}$ \\ E Liu ${ }^{2}$ \\ Guo-yan Mo² \\ Fang Huang' \\ 'China Key Laboratory of TCM \\ Resource and Prescription, Ministry \\ of Education, ${ }^{2}$ Department of \\ Pharmacy, Hubei University of \\ Chinese Medicine, Wuhan, Hubei, \\ People's Republic of China
}

\begin{abstract}
Malignant peripheral nerve sheath tumors (MPNSTs) are highly aggressive soft tissue neoplasms that are extremely rare and are frequently associated with neurofibromatosis type 1 patients. MPNSTs are typically fatal, and there is no effective treatment so far. In our previous study, we showed that flaccidoside II, one of the triterpenoid saponins isolated from Anemone flaccida Fr. Schmidt, has antitumor potential by inducing apoptosis. In the present study, we found that flaccidoside II inhibits proliferation and facilitates apoptosis in MPNST cell lines ST88-14 and S462. Furthermore, this study provides a mechanism by which the downregulation of heme oxygenase- 1 via extracellular signal-regulated kinase- $1 / 2$ and p38 mitogen-activated protein kinase pathways is involved in the apoptotic role of flaccidoside II. This study suggested the potential of flaccidoside II as a novel pharmacotherapeutic approach for MPNSTs.
\end{abstract}

Keywords: flaccidoside II, malignant peripheral nerve sheath tumors, apoptosis, MAPK, HO-1

\section{Introduction}

Neurofibromatosis (NF) is one of the most common human single-gene disorders, which clinically and genetically predisposes individuals to develop various benign and malignant tumors in the central and peripheral nervous systems, particularly in the brain. ${ }^{1,2} \mathrm{NF}$ is an autosomal dominant disorder, which results from mutations in either of the two tumor suppressor genes NF1 (neurofibromin) and NF2 (merlin). NF1 affects one in 3,500 individuals around the world, regardless of ethnicity and sex, ${ }^{3}$ and the most common occurrences are benign neurofibromas in NF1 patients, which include two subgroups: plexiform and dermal. ${ }^{4,5}$ Plexiform neurofibromas can progress to malignant sarcomas known as malignant peripheral nerve sheath tumors (MPNSTs), which are highly aggressive, resistant to conventional chemotherapy or surgical resection, and typically fatal, and are therefore a leading cause of mortality in NF1 patients. ${ }^{6,7}$ The absence of effective treatment for MPNSTs and NF1 unfolds the significance of understanding NF1 tumor development and seeking for effective therapeutic tools. Since the progress of MPNST is associated with additional genetic changes in oncogenes ${ }^{8}$ or loss of tumor suppressors, ${ }^{9}$ genetic modeling alongside NF1 has been considered to prevent MPNST development; ${ }^{10}$ although eventual resistance or tumor burden hinders its efficacy, ${ }^{11}$ new strategies for inhibition of both proliferation and survival are in need.
Correspondence: Guo-yan Mo Department of Pharmacy, Hubei University of Chinese Medicine, I Tan-Hua-Lin Road, Wuhan, Hubei 430065, People's Republic of China Email mogy.metabonomics@gmail.com

Fang Huang

China Key Laboratory of TCM Resource and Prescription, Ministry of Education, Wuhan, Hubei 430065, People's Republic of China

Email tracy.metabonomics@gmail.com (c) 2016 Han et al. This work is published and licensed by Dove Medical Press Limited. The full terms of this license are available at https://www.dovepress.com/terms.php
and incorporate the Creative Commons Attribution - Non Commercial (unported, v3.0) License (http://creativecommons.org/licenses/by-nc/3.0/). By accessing the work you BY
hereby accept the Terms. Non-commercial uses of the work are permitted without any further permission from Dove Medical Press Limited, provided the work is properly attributed. For permission hereby accept the Terms. Non-commercial uses of the work are permitted without any further permission from Dove Medical Press Limited, provided the work is properly attributed. For permission
for commercial use of this work, please see parazraphs 42 and 5 of our Terms (https:/wwww.dovepress com/terms php). 
The genus Anemone is widely distributed around the world, and $\sim 1 / 3$ species of Anemone are found in the People's Republic of China, which have been shown as valuable sources of diverse saponin compounds with useful biological properties. ${ }^{12-14}$ Anemone flaccida is widely used as Chinese folk medicine, and triterpenoid saponins, the main effective and characteristic ingredients of this genus, possess extensive biological activities, such as antitumor, antioxidative, and antibacterial properties. Several triterpenoid saponins were isolated, and their bioactivities have been reported. ${ }^{15-17}$ In our previous work, we have isolated five triterpenoid saponins from A. flaccida Fr. Schmidt: glycoside St-I4a (1), glycoside St-J (2), anhuienoside E (3), hederasaponin B (4), and flaccidoside II (5), which have shown antitumor effects by inducing apoptosis via cyclooxygenase-2/prostaglandin E2 (COX-2/PGE2) pathway. ${ }^{17,18}$ Accumulated evidence suggests that saponins have potential therapeutic effects on central nervous system disorders, such as stroke, Alzheimer's disease, and Parkinson's disease, while phytotherapy did not receive widespread attention. Here, we investigated the effect of triterpenoid saponin from A. flaccida on NF-related MPNSTs, and we found that flaccidoside II inhibited proliferation and facilitated apoptosis significantly in MPNST cell lines ST88-14 and S462.

The progress toward understanding the cellular and molecular alterations that are mediated by flaccidoside II may help in developing effective preventative and therapeutic strategies. The mammalian family of mitogen-activated protein kinases (MAPKs), including extracellular signalregulated kinase (ERK), p38 MAPK, and c-Jun N-terminal kinase (JNK), are evolutionarily conserved kinase modules that link extracellular signals to the machinery that controls fundamental cellular processes, including growth, proliferation, differentiation, and apoptosis, ${ }^{19}$ which play key roles in several steps of tumorigenesis, including cancer cell proliferation, migration, and invasion. ${ }^{20}$ Here, we assessed the effects of flaccidoside II on MAPK signaling pathways in our system and found that certain underlying pathways are involved in the antiapoptotic role of flaccidoside II.

We also investigated the role of flaccidoside II on heme oxygenase (HO)-1 and its antiapoptotic effect on MPNSTs. HO-1 is the inducible isoform of the first and rate-limiting enzyme of heme degradation. ${ }^{21}$ It has been reported that HO-1 not only protects against oxidative stress ${ }^{22}$ and apoptosis ${ }^{23}$ but also has potent anti-inflammatory functions. ${ }^{24,25}$ Furthermore, HO-1 also exerts facilitating effects on angiogenesis ${ }^{26}$ and on the proliferation of sarcoma and hepatoma cells in vivo. ${ }^{27}$ These findings suggest that the cytoprotective effect of HO-1 can modify the endogenous balance between apoptosis and proliferation toward an antiapoptotic and proproliferative status, which are relevant to oncogenesis, maintenance, and resistance to chemotherapy. In contrast, pharmacological agents that downregulate $\mathrm{HO}$ activity or HO-1 expression may become powerful tools for preventing the onset or progression of cancers and sensitize them to anticancer therapies. Coincidentally, we found in our experiments that flaccidoside II reduced the expression level and the activity of HO-1 in MPNST cell lines. Interestingly, our work also suggested that the roles of flaccidoside II on MAPK pathway might consequently affect the downstream HO-1, which ultimately triggers apoptosis in MPNST cell lines.

Collectively, the protective role of flaccidoside II on MPNSTs is revealed in this study. To our knowledge, this is the first study to report that flaccidoside II has antitumor capacity in the nervous system, which might represent a new insight for MPNST patient therapy and also reveal that the MAPK-HO-1 pathway is involved in the underlying mechanisms. This study might provide us clues for the development of new therapeutic drugs for NF1-associated MPNSTs.

\section{Materials and methods Cells and reagents}

The NF1-deficient human MPNST cell lines ST88-14 and S462 were cultured in Dulbecco's Modified Eagle's Medium (10\% fetal bovine serum, $1 \%$ penicillin-streptomycin, $1 \%$ L-glutamine, and $1 \%$ sodium pyruvate) and incubated at $37^{\circ} \mathrm{C}$ in a humidified atmosphere containing $10 \%$ carbon dioxide. No ethical committee approval was required for this set of experiments because the experiments were performed on commercially available cell lines. Dulbecco's Modified Eagle's Medium, fetal bovine serum, and tissue culture reagents were obtained from Thermo Fisher Scientific, Waltham, MA, USA. The following antibodies were used: anti-HO-1 (Epitomics, Burlingame, CA, USA), antiphospho-p38 (Cell Signaling Technology, Beverly, MA, USA), antiphosphoERK-1/2 (Cell Signaling Technology), antiphospho-JNK (Santa Cruz Biotechnology Inc., Dallas, TX, USA), anti-p38 (Cell Signaling Technology), anti-ERK-1/2 (Cell Signaling Technology), anti-JNK (Santa Cruz Biotechnology Inc.), and anti-GAPDH (Abcam, Cambridge, MA, USA). All other chemicals and reagents were of analytical grade.

\section{Cell proliferation}

MPNST cell lines ST88-14 and S462 were plated onto 96-well plates at a concentration of 10,000 cells/well in serum-containing growth medium. ${ }^{28}$ Viability of MPNST cell lines treated with triterpenoid saponin was assessed using CellTiter $96^{\circledR}$ Aqueous Non-Radioactive 
Cell Proliferation Assay (Promega Corporation, Fitchburg, WI, USA). After 24-hour incubation, cells were treated with carrier alone $(0.1 \%$ dimethyl sulfoxide [DMSO]), or flaccidoside II at the following gradient concentrations: $40.0 \mu \mathrm{mol} \mathrm{L}{ }^{-1}, 20.0 \mu \mathrm{mol} \mathrm{L}{ }^{-1}, 10.0 \mu \mathrm{mol} \mathrm{L}^{-1}, 5.0 \mu \mathrm{mol} \mathrm{L}{ }^{-1}$, and $2.50 \mu \mathrm{mol} \mathrm{L}^{-1}$. After incubation for 24 hours or 48 hours, the medium was carefully removed, replaced with fresh medium, $20 \mu \mathrm{L}$ of [3-(4,5-dimethylthiazol-2-yl)-5-(3carboxymethoxy-phenyl)-2-(4-sulfophenyl)-2H-tetrazolium, inner salt] (MTS) was added to each well, and incubated at $37^{\circ} \mathrm{C}$ for 2 hours. The amount of proliferation was quantified by MTS assay using Cell titer 96 proliferation kit (Promega Corporation), and absorbance at $490 \mathrm{~nm}$ was read in a Spectramax M2 plate reader (Molecular Devices LLC, Sunnyvale, CA, USA) at the designated time points. ${ }^{29}$ The percentage of viable cells at each condition was calculated by dividing the absorbance (A490) of treated cells by that of control cells. The half maximal inhibitory concentration $\left(\mathrm{IC}_{50}\right)$ was determined from the graph of cell viability (\%) vs concentrations. Each experiment was performed in quadruplicate and repeated three times independently.

\section{Apoptosis assay}

The ST88-14 and S462 cells were plated in serum-containing growth medium in $60 \mathrm{~mm}$ culture dishes at a seeding density of $5 \times 10^{5}$ cells/dish. Cells were treated with carrier alone ( $0.1 \%$ DMSO) or flaccidoside II at the following gradient concentrations: $40.0 \mu \mathrm{mol} \mathrm{L}^{-1}, 20.0 \mu \mathrm{mol} \mathrm{L}^{-1}, 10.0 \mu \mathrm{mol} \mathrm{L}^{-1}$, $5.0 \mu \mathrm{mol} \mathrm{L}{ }^{-1}$, and $2.50 \mu \mathrm{mol} \mathrm{L} \mathrm{L}^{-1}$ for 24 hours or 48 hours. After incubation for designated times, apoptosis was assayed by using an Annexin V-PI staining kit by following the manufacturer's procedure (KeyGen Biotechnology Co. Ltd., Nanjing, People's Republic of China) as described earlier. ${ }^{17,30}$ The apoptotic rate was then detected by flow cytometry with the standardized program of the instrument (FACSCalibur; BD Biosciences, San Jose, CA, USA). Experiments were performed in four duplicates for each condition in each experiment, and a minimum of 500 cells was counted in each case. Assays were repeated three times independently.

\section{Protein isolation and Western blotting}

Protein extracts were prepared as previously described from MPNST cell lines ST88-14 and S462 growing in log phase in serum-containing growth medium. Cells were treated with $40.0 \mu \mathrm{mol} \mathrm{L}^{-1}$ flaccidoside II for 48 hours. Nuclear and cytosolic fractions of proteins were obtained by a commercially available nuclear extraction kit (Panomics, Inc., Fremont, CA, USA). ${ }^{31}$ Then, cells were rinsed twice in ice-cold phosphatebuffered saline ( $\mathrm{pH}$ 7.5), and cell lysates were prepared for
Western blotting analysis by using whole cellular protein extraction kits (Santa Cruz Biotechnology Inc.). Protein concentration was determined using BCA kit (Pierce, Rockford, IL, USA). The cell lysate with certain amount of protein (20-50 $\mu \mathrm{g}$ ) was first separated by $10 \%$ sodium dodecyl sulfate polyacrylamide gel electrophoresis gels and then electrically transferred to polyvinylidene difluoride membrane (EMD Millipore, Billerica, MA, USA). Detected proteins were incubated with specific antibodies overnight, immunoreactive bands were then visualized by an enhanced chemiluminescence plus detection kit (GE Biosciences, GE Healthcare Bio-Sciences, PA, USA) and exposed to CL-XPosure film. Protein bands were quantitatively analyzed by Kodak Digital Science 1D software (Eastman Kodak Company, New Haven, CT, USA) and were expressed as sum optical density. ${ }^{32}$

\section{$\mathrm{HO}$ enzyme activity assay}

HO enzyme activity was determined as described previously. ${ }^{33}$ The reaction was carried out in the dark for 1 hour at $37^{\circ} \mathrm{C}$ then stopped by placing the tubes on dry ice, and bilirubin was extracted with chloroform. The amount of extracted bilirubin was estimated by the difference in absorbance between $463 \mathrm{~nm}$ and $520 \mathrm{~nm}$ by using a scanning spectrophotometer. The $\mathrm{HO}$ activity was calculated as pmol $\mathrm{mg}^{-1} \mathrm{~h}^{-1}$ and presented as fold change.

\section{Statistical analysis}

Statistical analysis among the various treatment groups in cell proliferation and apoptosis studies was performed by Prism (GraphPad Software, Inc., La Jolla, CA, USA). The one-way or two-way ANOVA procedure followed by least significant difference post hoc tests and Student's $t$-test was used to determine the statistical significance of differences of the means. $P<0.05$ was considered statistically significant. The data were expressed as mean \pm standard deviation $(\mathrm{SD})$.

\section{Results}

\section{Flaccidoside II inhibits proliferation and facilitates apoptosis in MPNST cell lines}

First, we investigated the effect of triterpenoid saponin from the A. flaccida on NF by performing flaccidoside II in NF1related human MPNST cell lines ST88-14 and S462. The reason why we applied flaccidoside II here as a representative triterpenoid from compounds $1-5$ is based on its "comprehensive" antitumor effect. We have assessed the antitumor effects of different triterpenoid saponins on several cancer cell models, including human BEL-7402 and HepG2 hepatoma cell lines, as well as the model of HeLa cells treated 
with lipopolysaccharide. According to our previous data, flaccidoside II has stronger antitumor effects comparing to other triterpenoid saponins in all the cell models we have measured. ${ }^{17}$ We measured the effect of flaccidoside II on MPNST cell proliferation by MTS assay. ST88-14 and S462 cells were treated with flaccidoside II at the following five gradient concentrations: $40.0 \mu \mathrm{mol} \mathrm{L}-1,20.0 \mu \mathrm{mol} \mathrm{L}-1,10.0 \mu \mathrm{mol} \mathrm{L}-1$, $5.0 \mu \mathrm{mol} \mathrm{L}^{-1}$, and $2.50 \mu \mathrm{mol} \mathrm{L}-1$ for 24 hours or 48 hours. As shown in Figure 1A and B, treatment of flaccidoside II at varying doses on ST88-14 and S462 cells showed that cytotoxicity induced by flaccidoside II was dose and time dependent with $90 \%-95 \%$ inhibition achieved at maximum concentration of $40.0 \mu \mathrm{mol} \mathrm{L}{ }^{-1}$ after 48 hours of treatment.

We then characterized apoptosis of ST88-14 and S462 cells when treated with flaccidoside II that was demonstrated by Annexin V-FITC/PI flow cytometry assay. The results from ST88-14 and S462 cells are illustrated in Figure $2 \mathrm{~A}$ and $\mathrm{B}$, respectively. Consistent with the cell growth inhibition effect of flaccidoside II, the percentage of both early (18.7\%) and late apoptotic cells (32.8\%) for flaccidoside II treatment in ST88-14 and S462 cells significantly increased compared to controls. The apoptosis of ST88-14 and S462 cells induced by flaccidoside II was also confirmed by Western blotting measurements on the expression of cleaved PARP and cleaved caspase 3 at the gradient performance of flaccidoside II for 24 hours and 48 hours (Figure 2C-F). These data suggested that flaccidoside II inhibits proliferation in MPNST ST88-14 and S462 cells via inducing apoptosis.
Flaccidoside II-mediated signal activates P38 MAPK and inhibits ERK-I/2 via phosphorylation

To gain insight into the mechanism of action for flaccidoside II in MPNST tumorigenesis, we evaluated the MAPK signaling pathways. We and others have shown that triterpenoid saponins inhibit the COX-2/PGE2 pathway in cancer cells, which contribute their apoptotic effects, and the upstream MAPK-related inflammatory pathways play important roles for prevention and treatment of cancer by triterpenoids. ${ }^{34-36}$ Here, we wished to check whether flaccidoside II can modulate the activation of ERK-1/2, JNK, and p38 MAPK. The ST88-14 and S462 cells were treated with either $40.0 \mu \mathrm{mol} \mathrm{L}{ }^{-1}$ flaccidoside II or DMSO for 48 hours. Following treatment, the phosphorylation statuses and total levels of p38, JNK, and ERK-1/2 were measured by Western blot. As shown in Figure $3 \mathrm{~A}-\mathrm{C}$, the application of flaccidoside II increased the phosphorylation of p38 MAPK and reduced ERK-1/2 phosphorylation, while there were no significant changes in the phosphorylation of JNK, as well as the total levels of p38, JNK, and ERK-1/2 (Figure 3A-D). In addition, we found that the COX-2 protein expression level was markedly downregulated by flaccidoside II treatment, as shown in Figure $3 \mathrm{~A}$ and E.

\section{Flaccidoside II-mediated signaling downregulates the expression and activation of antiapoptotic $\mathrm{HO}-\mathrm{I}$}

As shown in our previous work, flaccidoside II inhibits the COX-2/PGE2 pathway, which has key roles in the hallmarks

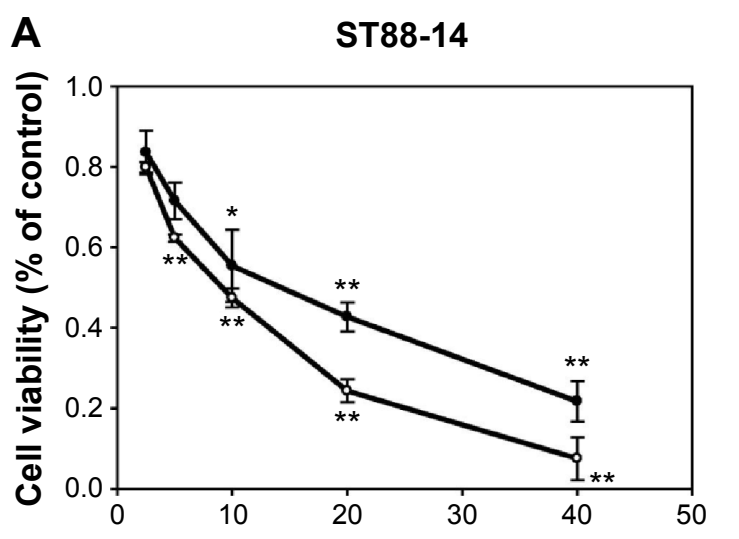

Concentration of flaccidoside II $(\mu \mathrm{mol} \mathrm{L}-1)$

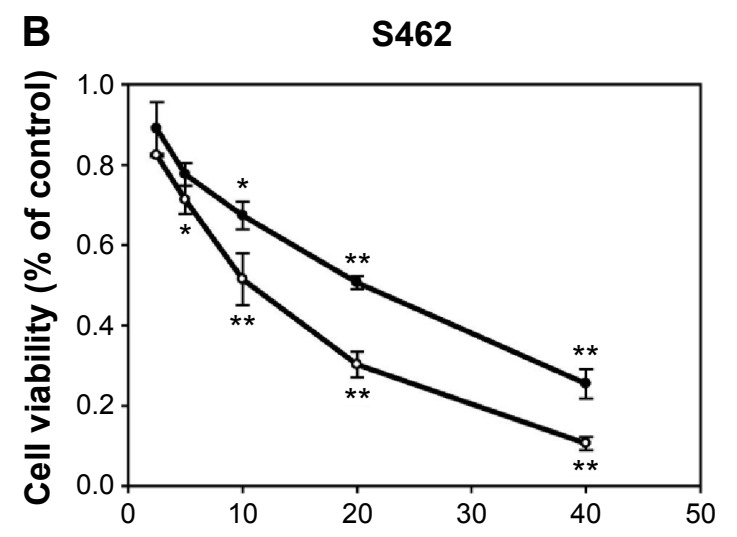

\section{Concentration of flaccidoside II $\left(\mu \mathrm{mol} \mathrm{L} \mathrm{L}^{-1}\right)$}

24 hours -0 hours

Figure I Flaccidoside II inhibits proliferation in MPNST cell lines.

Notes: NFI-related human MPNST cell lines ST88-I4 (A) and S462 (B) were treated with flaccidoside II at five gradient concentrations: $40.0 \mu$ mol L-1, $20.0 \mu$ mol $L^{-1}$,

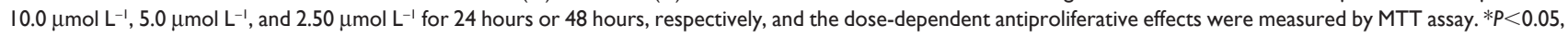
$* * P<0.01$ vs control (mean $\pm S D$ ). Each experiment was repeated at least three times, except specifically stated.

Abbreviations: MPNST, malignant peripheral nerve sheath tumor; NFI, neurofibromatosis type I; SD, standard deviation. 
A

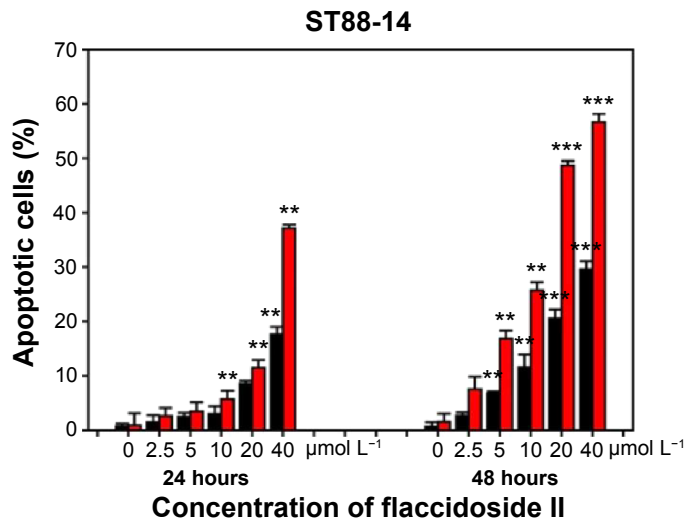

B

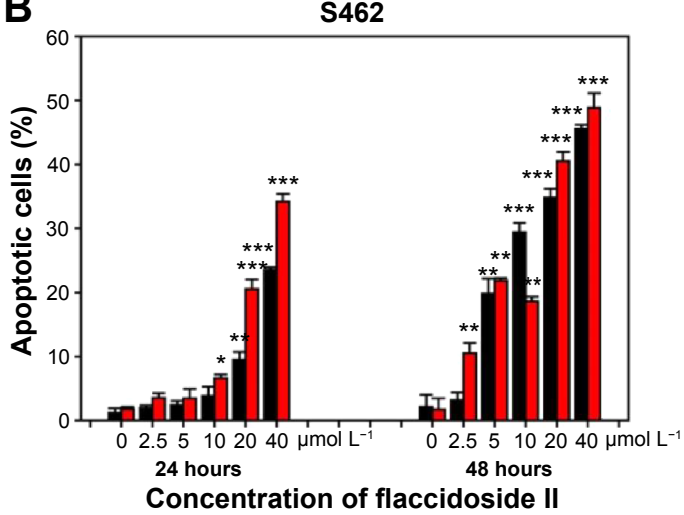

Early apoptotic $\square$ Late apoptotic

C

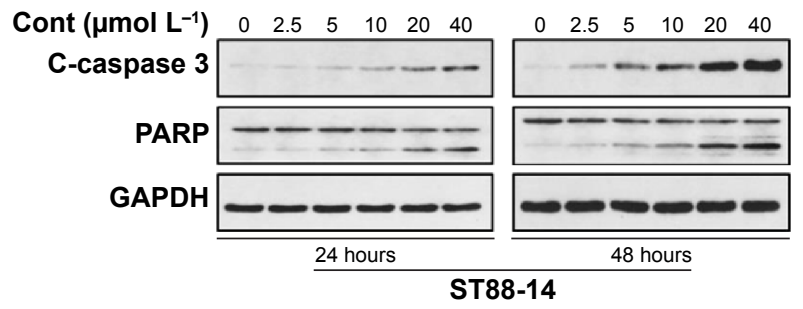

$\mathbf{E}$

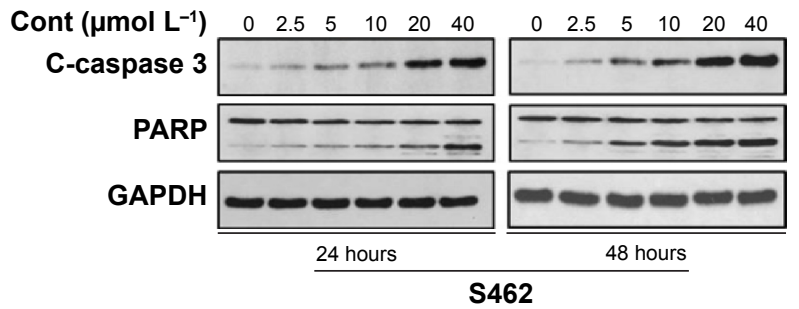

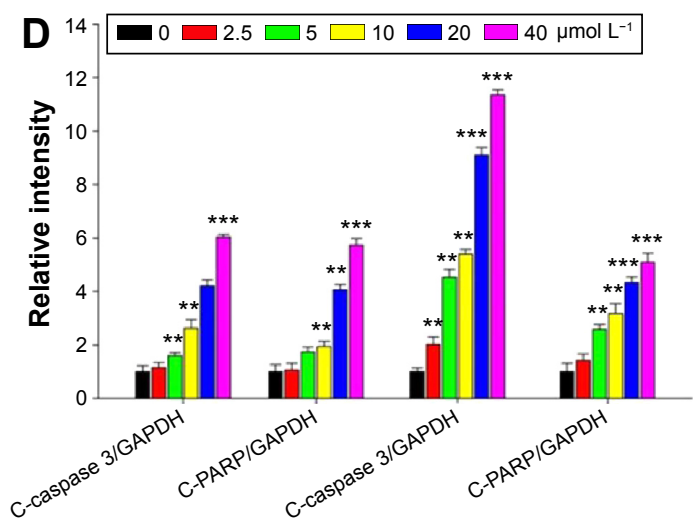

24 hours 48 hours

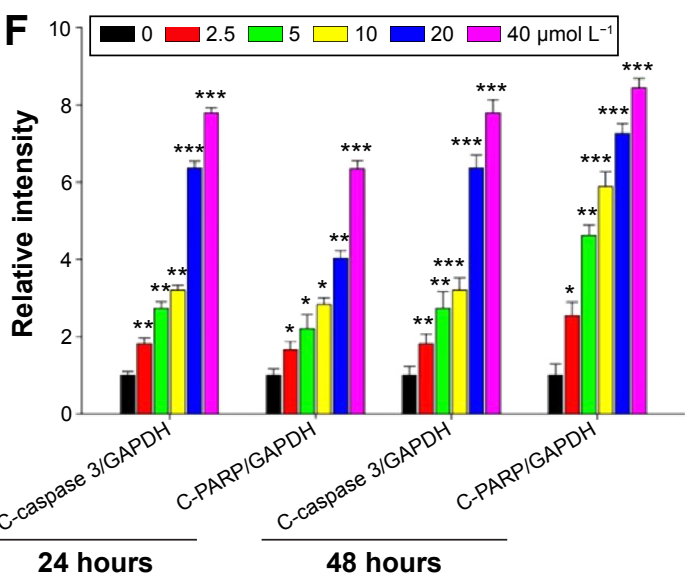

Figure 2 Flaccidoside II facilitates apoptosis in MPNST cell lines.

Notes: NFI-related human MPNST cell lines ST88-14 (A) and S462 (B) were treated with flaccidoside II at five gradient concentrations: $40.0 \mu$ mol L-1, $20.0 \mu$ mol $L^{-1}$, $10.0 \mu \mathrm{mol} \mathrm{L}-1,5.0 \mu \mathrm{mol} \mathrm{L}-1$, and $2.50 \mu \mathrm{mol} \mathrm{L}^{-1}$ for 24 hours or 48 hours, respectively, and the early and late apoptotic rates were determined by Annexin V-PI, staining and flow cytometry for quantification. $* P<0.05, * * P<0.01$, and $* * * P<0.001$ vs control (mean $\pm S D$ ). (C) The ST88-14 cell lines were treated with five gradient concentrations of flaccidoside II for 24 hours or 48 hours, then the C-caspase 3, total PARP, and cleaved PARP level were determined by Western blotting, GAPDH was used as loading control. (D) Quantitative analysis of the blots in (C). (E) The S462 cell lines were treated with five gradient concentrations of flaccidoside II for 24 hours or 48 hours, then the C-caspase 3, total PARP, and cleaved PARP level were determined by Western blotting. (F) Quantitative analysis of the blots in (E). Data represented as mean \pm SD of four independent experiments. $* P<0.05$, $* * P<0.01$, and $* * * P<0.00$ I vs control.

Abbreviations: MPNST, malignant peripheral nerve sheath tumor; NFI, neurofibromatosis type I; Cont, control; SD, standard deviation.

of cancer and adaptation to the tumor microenvironment. HO-1 has been reported to play a central role in protecting cells against oxidative stress and apoptosis as a prime cellular defense mechanism. ${ }^{24}$ Serendipitously, we found in our experiment that HO-1 protein levels were markedly decreased in ST88-14 and S462 cells after treatment with flaccidoside II as shown in Figure 4A, and HO-1 has also shown to be inactivated by flaccidoside II (Figure 4B). In contrast, we also evaluated how the growth inhibitory functions of flaccidoside II can be affected if we overexpress 

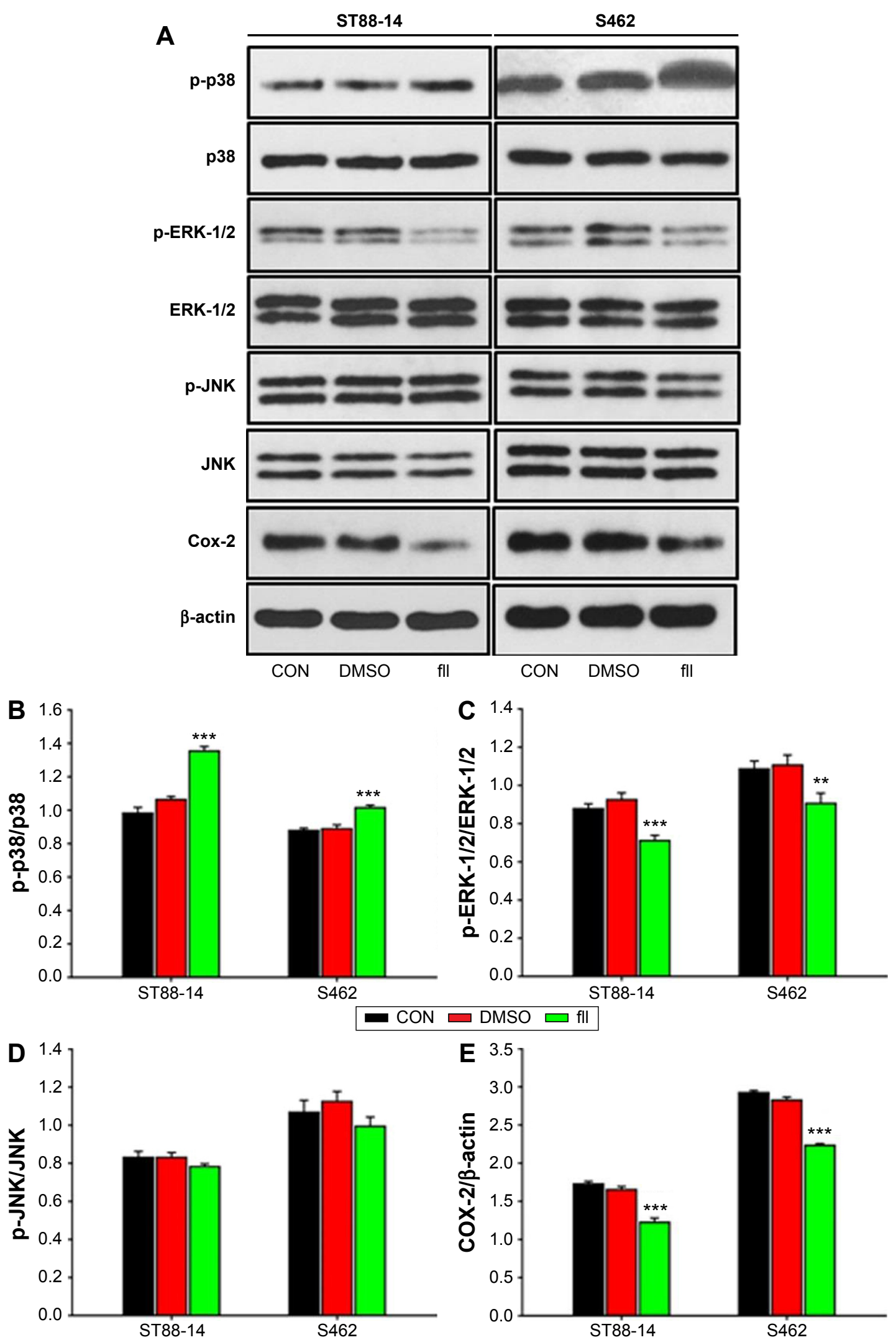

Figure 3 Flaccidoside II activates P38 MAPK and inhibits ERK-I/2 via phosphorylation, but not JNK pathway.

Notes: The ST88-I4 and S462 cells were treated with either $40.0 \mu \mathrm{mol} \mathrm{L^{-1 }}$ flaccidoside II or DMSO for 48 hours, and the protein expression levels of phosphorylation and total P38 MAPK, ERK-1/2, and JNK as well as the protein expression level of COX-2 were measured by Western blotting, and $\beta$-actin was used as loading control (A). (B-E) Quantitative analyses of the blots in (A). Data represent the mean \pm SD of four independent experiments. $* P<0.05$, $* * P<0.0$ I, and $* * * P<0.00$ I vs control.

Abbreviations: MAPK, mitogen-activated protein kinase; ERK-I/2, extracellular signal-regulated kinase I/2; JNK, c-Jun N-terminal kinase; DMSO, dimethyl sulfoxide; COX-2, cyclooxygenase-2; CON, control; fll, flaccidoside II; p-JNK, phosphor-JNK; SD, standard deviation. 
A
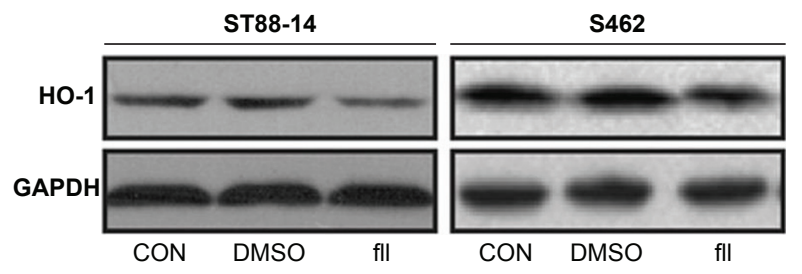

C
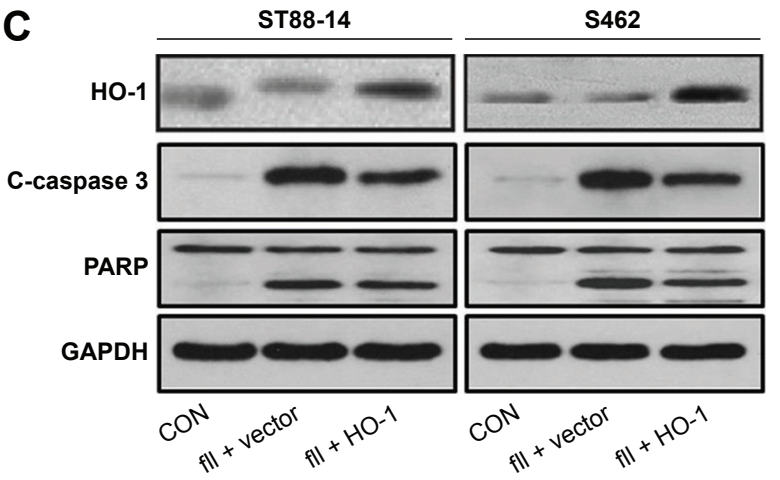
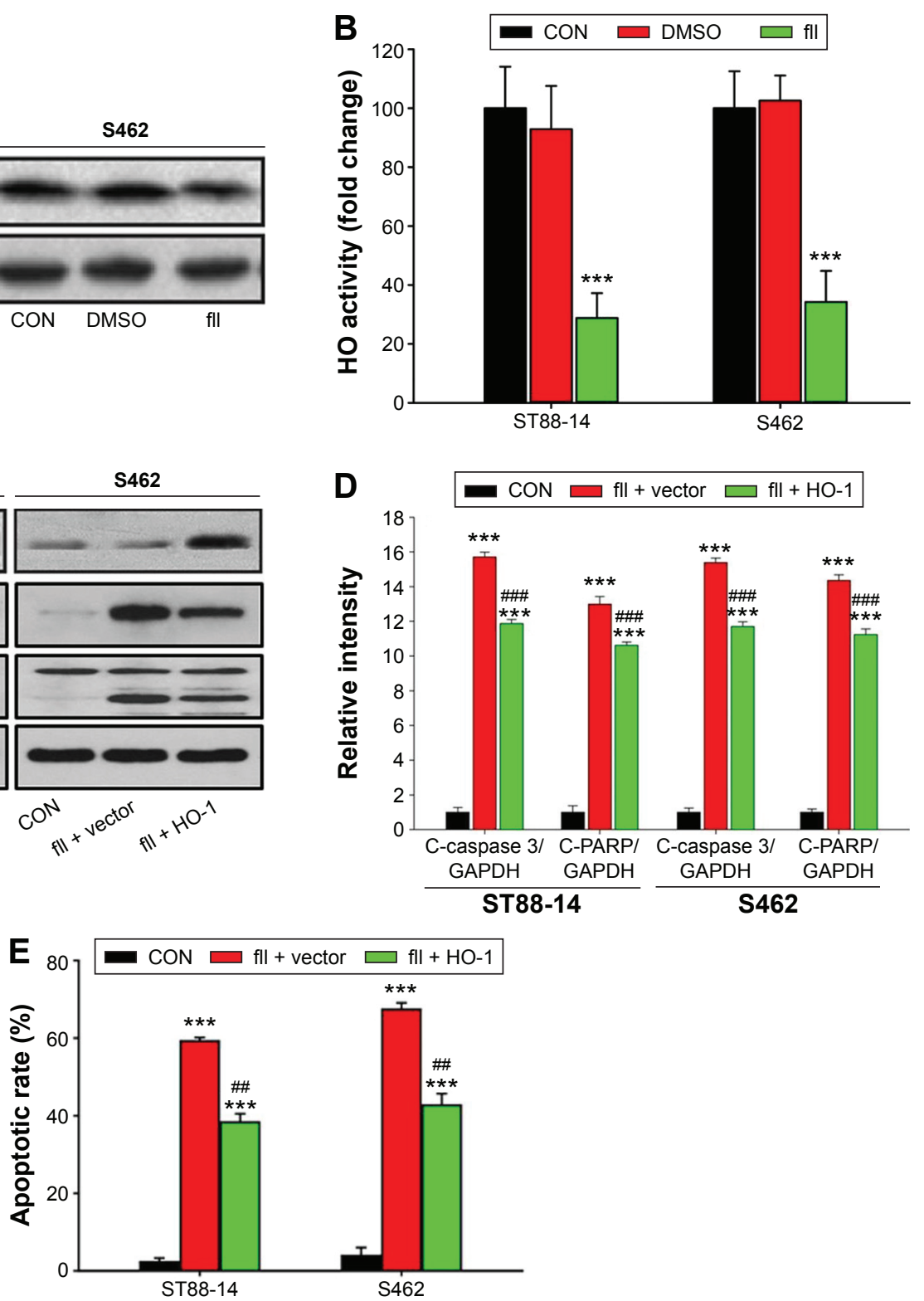

Figure 4 Flaccidoside II downregulates the expression and activation of antiapoptotic HO-I, which is involved in the apoptotic facilitation of flaccidoside II.

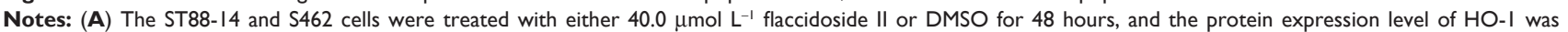
measured by Western blotting, which was normalized to GAPDH. (B) The HO activity was measured in ST88-14 and S462 cells at 48 hours after treatment of these cells with $40.0 \mu \mathrm{mol} \mathrm{L} \mathrm{L}^{-1}$ flaccidoside II or DMSO. (C) The ST88-I4 and S462 cells were treated with $40.0 \mu \mathrm{mol}^{-1}$ flaccidoside II with the transfection of either vector plasmid as a negative control or full-length HO-I plasmid. The expression of HO-I, C-caspase 3, total PARP, and cleaved PARP were determined by Western blotting, and GAPDH was used as loading control (C). (D) Quantitative analysis of the apoptosis markers cleaved caspase 3 and cleaved PARP in (C). (E) The apoptotic rates of ST88-I4 and S462 cells were determined by Annexin V-PI staining and flow cytometry for quantification. Each bar represents the mean \pm SD of four independent experiments. $* * * P<0.00 \mathrm{I}$ vs control, ${ }^{\# P}<0.01, \ldots P<0.00$ I vs fll + vector.

Abbreviations: HO-I, heme oxygenase I; DMSO, dimethyl sulfoxide; CON, control; fll, flaccidoside II; SD, standard deviation.

HO-1 in MPNST cells. We treated MPNST cells ST88-14 and S462 with flaccidoside II in the absence or presence of HO-1 overexpressed plasmid transfection and then compared the apoptotic rate of the transfected cells with nontransfected cells after 48 hours. The overexpression of HO-1 was confirmed by Western blotting (Figure 4C), and then the apoptosis of the cells was measured by flow cytometry and Western blotting of apoptosis marker PARP and caspase 3 . As shown in Figure 4C-E, flaccidoside II increased apoptosis of ST88-14 and S462 cells; while flaccidoside II could not induce apoptosis with HO-1 overexpression up to the similar level as observed in nontransfected cells.

\section{HO-I downregulation induced by flaccidoside II is mediated by ERK-I/2 and p38 MAPK pathways}

Several signaling pathways are reported to be involved in the regulation of HO- $1,{ }^{37}$ including PKC, ${ }^{38} \mathrm{PI} 3 \mathrm{~K} / \mathrm{AKT},{ }^{39}$ and 
A

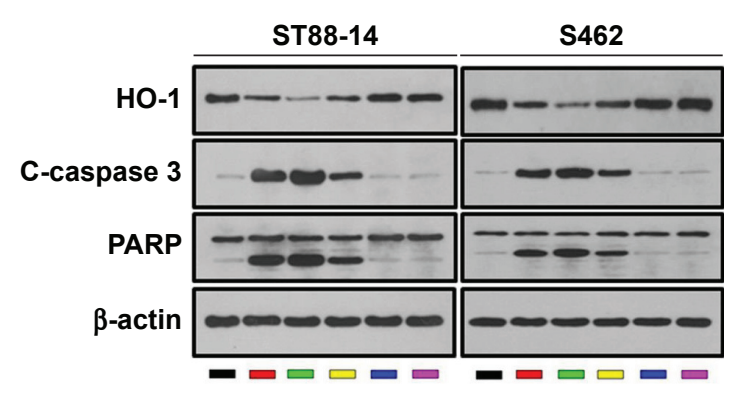

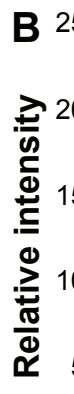

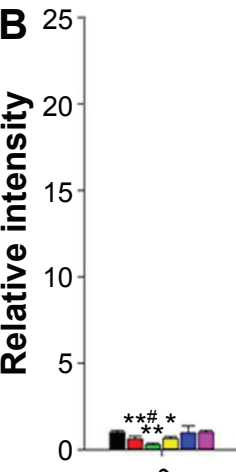

C

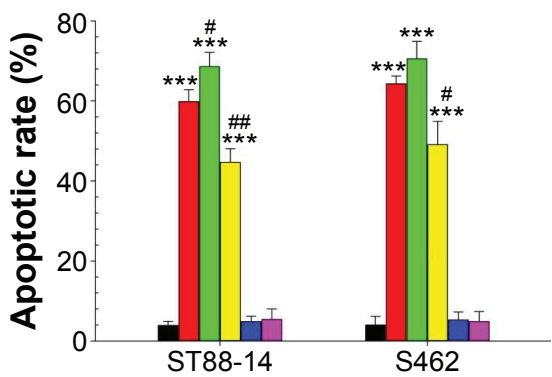

D

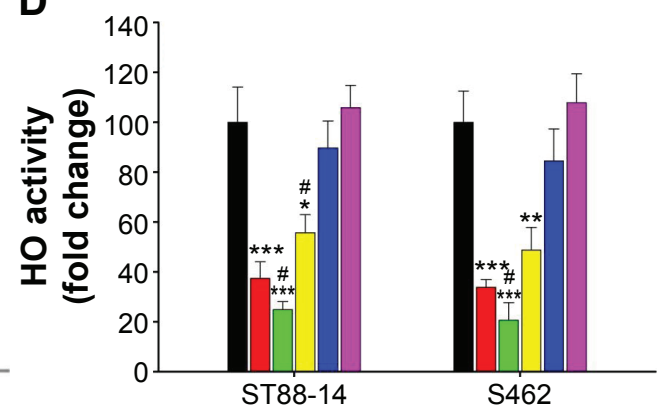

Figure 5 HO-I downregulation induced by flaccidoside II is mediated by ERK-I/2 and P38 MAPK pathways.

Notes: (A-C) The ST88-I 4 and S462 cells were treated with $40.0 \mu \mathrm{mol} \mathrm{L}^{-1}$ flaccidoside II with performance of ERK-1/2 inhibitors PD98059 or P38 inhibitor SB203580, and the protein expression level of HO-I, C-caspase 3, total PARP, and cleaved PARP were measured by Western blotting, and $\beta$-actin was used as loading control (A). (B) Quantitative analysis of the blots in (A). (C) The apoptotic rates of cells were determined by Annexin V-PI staining and flow cytometry for quantification. (D) HO activity was measured in ST88-I 4 and S462 cells at 48 hours treatment of $40.0 \mu \mathrm{mol} \mathrm{L-1}$ flaccidoside II with/without the performance of ERK-I/2 inhibitors PD 98059 or p38 inhibitor SB203580, respectively. Each bar represents the mean \pm SD of at least four independent experiments. $* P<0.05$, $* * P<0.0$ I, and $* * * P<0.001$ vs control; ${ }^{*} P<0.05$ and $\# P<0.0$ l vs fll treatment.

Abbreviations: HO-I, heme oxygenase I; ERK-I/2, extracellular signal-regulated kinase I/2; MAPK, mitogen-activated protein kinase; fll, flaccidoside II; SD, standard deviation.

$\mathrm{MAPK}^{40}$ pathways. Here, we investigated whether the upstream MAPK signaling pathway is involved in flaccidoside II-mediated HO-1 regulation, we next tested the effects of ERK-1/2 inhibitors PD98059 and p38 inhibitor SB203580 on HO-1 and MPNST apoptosis in the presence of flaccidoside II. Interestingly, we have found that the inhibitors alone did not change the apoptosis on basal level, while the application of ERK-1/2 inhibitors PD98059 further facilitated the apoptotic rate of ST88-14 and S462 cells with treatment of flaccidoside II; and it was reduced by p38 inhibitor SB203580 verified by Western blotting of apoptosis marker cleaved PARP and cleaved caspase 3 (Figure 5A-C). Meanwhile, PD98059 decreased the expression/activity of HO-1, while SB203580 increased them contrarily, although the effects were not significant when flaccidoside II was absent (Figure 5A, $\mathrm{B}$, and $\mathrm{D})$. These data suggested that ERK-1/2 and $\mathrm{p} 38$ MAPK pathways are involved in the downregulation of HO-1 mediated by flaccidoside II.

\section{Discussion}

The MPNSTs are aggressive, chemoresistant soft-tissue tumors with poor 5-year survival that arise in patients with NF1 or sporadically. By using NF1-related human MPNST cell lines, we sought to discover new compounds isolated from natural plants, which have potential therapeutic effects on MPNST by comparing the chemical structures with the data from an unbiased high-throughput chemical library screening approach (data not shown here). In this way, we found the promising compounds such as flaccidoside II, one of the triterpenoid saponins from A. flaccida Fr. Schmidt. Here, we performed cell proliferation assay and flow cytometry to confirm that flaccidoside II inhibits proliferation and facilitates apoptosis in NF1-associated MPNSTs cell lines ST88-14 and S462. Importantly, this is the first study to report that a compound from natural plants has antitumor capacity, which might represent a new insight for MPNST patient therapy. 
The use of medicinal plants or their active components is becoming an increasingly attractive approach for the treatment of various inflammatory disorders and cancer. Flaccidoside II, as a bidesmosidic triterpene saponin isolated from Chinese folk medicine, has been synthesized and reported in previous research. ${ }^{13,41}$ However, very little is known about the function of flaccidoside II and its therapeutic potentials for human diseases. Resistance against apoptosis plays a central role in the cancer-supportive environment and provides significant rejection to the cytotoxicity of chemotherapy. ${ }^{42}$ Our previous work has revealed the antiapoptotic role of flaccidoside II in cancer cell lines. In the present work, we investigated its effect on NF1-associated MPNSTs in the central and peripheral nervous systems. Combining our previous work ${ }^{18}$ with the data from highthroughput chemical library screening, it is not surprising to observe the roles on proliferation inhibition and apoptosis facilitation of flaccidoside II in MPNST cell lines, which could be due to its structure in which an 1-rhamnopyranose links to other sugars at the end of glycosidic chain in C-3 position. ${ }^{17}$

Furthermore, we define the mechanism by which flaccidoside II can mediate novel growth inhibitory signals in MPNST cells. Since we have reported in our previous work that the expression of COX-2 is inhibited by performing triterpenoid saponins, including flaccidoside $\mathrm{II},{ }^{17}$ and MAPK signaling pathways are known to be involved in the regulation of COX-2 expression, ${ }^{43,44}$ we sought to investigate whether MAPK signaling pathways participate in the underlying mechanisms of antiapoptotic role of flaccidoside II. We measured the activation and expression status of major MAPK family members, including ERK, p38, and JNK. Our data suggested that flaccidoside II activates p38 MAPK and inhibits ERK-1/2 via phosphorylation, while it has no significant effect on the phosphorylation of JNK and the total expression levels of ERK, p38, and JNK. Since MAP kinase signaling pathways represent a potential target for therapeutic intervention for cancers, ${ }^{45}$ here we revealed that flaccidoside II facilitates apoptosis in MPNSTs via regulating the activation of p38 MAPK and ERK-1/2, but not JNK pathway, which gave us a hint that flaccidoside II could be considered as a novel pharmacotherapeutic approach to MPNSTs based on its regulation of MAPK pathways.

$\mathrm{HO}-1$ is one of the cytoprotective enzymes in response to various stimuli, including cellular oxidative stress and inflammation. HO-1 is highly induced in various disease states, including neurodegenerative diseases and cancer.
Several lines of evidences have suggested the protective role of HO-1 in oxidative stress-induced neuronal injury ${ }^{46}$ and the expression of HO-1 may be regulated by oxidative stresses in a coordinated manner with tau, which plays a pivotal role in the cytoprotection of neuronal cells. ${ }^{47}$ However, in contrast, accumulating reports have supported that HO-1 participates in carcinogenesis and tumor progression by promoting proliferation and survival, inducing angiogenesis through regulating expression of angiogenic factors, and modulating tumor microenvironment as well. ${ }^{48}$ The protumorigenic role of HO-1 renders its potential as a molecular target for cancer therapy, while very little is known about the role of HO-1 on tumors in nervous system such as NF1-associated MPNSTs. In this study, we have shown that flaccidoside II downregulates the expression and the activation of HO-1, which is involved in the mechanisms of apoptotic facilitation of flaccidoside II. Furthermore, several reports demonstrated that $\mathrm{p} 38$-MAPK $,{ }^{49} \mathrm{ERK},{ }^{49,50} \mathrm{PI} 3 \mathrm{~K},{ }^{51}$ and PKC- $\delta^{52}$ are involved in HO-1 expression/activity. Signaling mechanisms involved in HO-1 induction may depend on cell types and inducers. Consistent with previous reports that MAPK pathways are important in modulating the HO-1 expression, ${ }^{49,53}$ we found in our experiments that ERK-1/2 and p38 MAPK pathways are upstream of HO-1 and involved in the downregulation of HO-1 mediated by flaccidoside II. Interestingly and importantly, we noticed in our experiments that the inhibitors alone did not change the HO-1 expression/activity on basal level, while the decrease in HO-1 expression/activity induced by flaccidoside II is regulated by ERK-1/2 and p38 MAPK inhibitors. These data strongly suggested the therapeutic potentials of ERK-1/2 and p38 MAPK pathways in HO-1 downregulation induced by flaccidoside II due to the specific effects at pathological state. There is no doubt that the in vivo verification of the phytotherapeutic potential of flaccidoside II on NF1-associated MPNSTs via the MAPKHO-1 pathway such as in MPNST mouse model would be necessary in further research, although the MPNST cell lines ST88-14 and S462 are reliable for exploring the molecular mechanisms of the apoptotic effect of flaccidoside II.

Taken together, this study supports the view that flaccidoside II inhibits proliferation and facilitates apoptosis in NF1-associated MPNST cell lines, which renders its potential as a novel pharmacotherapeutic approach for MPNSTs. Furthermore, downregulation of HO-1 is required for the apoptotic role of flaccidoside II in MPNST cell lines ST88-14 and S462, and ERK-1/2 and p38 MAPK pathways are the upstream modulators for the process. 


\section{Acknowledgments}

This research was funded by the National Natural Science Foundation of China (grant no 31000158), the National Science Foundation of Hubei Province of China (grant no 2013CFA040), and Wuhan Science and Technology Talent cultivation plan (grant no 2014070404010216).

\section{Disclosure}

The authors report no conflicts of interest in this work.

\section{References}

1. Goldberg NS, Collins FS. The hunt for the neurofibromatosis gene. Arch Dermatol. 1991;127(11):1705-1707.

2. Crawford AH, Schorry EK. Neurofibromatosis update. JPediatr Orthop. 2006;26(3):413-423.

3. Wallace MR, Marchuk DA, Andersen LB, et al. Type 1 neurofibromatosis gene: identification of a large transcript disrupted in three NF1 patients. Science. 1990;249(4965):181-186.

4. Lin AL, Gutmann DH. Advances in the treatment of neurofibromatosisassociated tumours. Nat Rev Clin Oncol. 2013;10(11):616-624.

5. Zwarthoff EC. Neurofibromatosis and associated tumour suppressor genes. Pathol Res Pract. 1996;192(7):647-657.

6. Zoller M, Rembeck B, Akesson HO, Angervall L. Life expectancy, mortality and prognostic factors in neurofibromatosis type 1. A twelveyear follow-up of an epidemiological study in Goteborg, Sweden. Acta Derm Venereol. 1995;75(2):136-140.

7. Duong TA, Sbidian E, Valeyrie-Allanore L, et al. Mortality associated with neurofibromatosis 1: a cohort study of 1895 patients in 1980-2006 in France. Orphanet J Rare Dis. 2011;6:18.

8. Cichowski K, Shih TS, Schmitt E, et al. Mouse models of tumor development in neurofibromatosis type 1. Science. 1999;286(5447): 2172-2176.

9. Endo M, Kobayashi C, Setsu N, et al. Prognostic significance of p14ARF, p15INK4b, and p16INK4a inactivation in malignant peripheral nerve sheath tumors. Clin Cancer Res. 2011;17(11):3771-3782.

10. Jessen WJ, Miller SJ, Jousma E, et al. MEK inhibition exhibits efficacy in human and mouse neurofibromatosis tumors. J Clin Invest. 2013; 123(1):340-347.

11. Widemann BC. Current status of sporadic and neurofibromatosis type 1-associated malignant peripheral nerve sheath tumors. Curr Oncol Rep. 2009;11(4):322-328.

12. Zhao L, Chen WM, Fang QC. Triterpenoid saponins from Anemone flaccida. Planta Med. 1990;56(1):92-93.

13. Zhao L, Chen WM, Fang QC. Two new oleanane saponins from Anemone flaccida. Planta Med. 1991;57(6):572-574.

14. Ye WC, Zhang QW, Zhao SX, Che CT. Four new oleanane saponins from Anemone anhuiensis. Chem Pharm Bull. 2001;49(5):632-634.

15. Chen X, Lu J, He W, et al. Antiperoxidation activity of triterpenoids from rhizome of Anemone raddeana. Fitoterapia. 2009;80(2):105-111.

16. Wang XY, Chen XL, Tang HF, Gao H, Tian XR, Zhang PH. Cytotoxic triterpenoid saponins from the rhizomes of Anemone taipaiensis. Planta Med. 2011;77(13):1550-1554.

17. Han LT, Fang Y, Li MM, Yang HB, Huang F. The antitumor effects of triterpenoid saponins from the Anemone flaccida and the underlying mechanism. Evid Based Complement Alternat Med. 2013;2013: 517931.

18. Han LT, Li J, Huang F, Yu SG, Fang NB. Triterpenoid saponins from Anemone flaccida induce apoptosis activity in HeLa cells. J Asian Nat Prod Res. 2009;11(2):122-127.

19. Dhillon AS, Hagan S, Rath O, Rath O, Kolch W. MAP kinase signalling pathways in cancer. Oncogene. 2007;26(22):3279-3290.

20. Kim EK, Choi EJ. Pathological roles of MAPK signaling pathways in human diseases. Biochim Biophys Acta. 2010;1802(4):396-405.
21. Tenhunen R, Marver HS, Schmid R. The enzymatic conversion of heme to bilirubin by microsomal heme oxygenase. Proc Natl Acad Sci US A. 1968;61(2):748-755.

22. Pae HO, Jeong SO, Koo BS, Ha HY, Lee KM, Chung HT. Tranilast, an orally active anti-allergic drug, up-regulates the anti-inflammatory heme oxygenase-1 expression but down-regulates the pro-inflammatory cyclooxygenase- 2 and inducible nitric oxide synthase expression in RAW264.7 macrophages. Biochem Biophys Res Commun. 2008; 371(3):361-365.

23. Lee BW, Chun SW, Kim SH, et al. Lithospermic acid B protects betacells from cytokine-induced apoptosis by alleviating apoptotic pathways and activating anti-apoptotic pathways of Nrf2-HO-1 and Sirt1. Toxicol Appl Pharmacol. 2011;252(1):47-54.

24. Ryter SW, Alam J, Choi AM. Heme oxygenase-1/carbon monoxide: from basic science to therapeutic applications. Physiol Rev. 2006;86(2): 583-650.

25. Willis D, Moore AR, Frederick R, Willoughby DA. Heme oxygenase: a novel target for the modulation of the inflammatory response. Nat Med. 1996;2(1):87-90.

26. Jozkowicz A, Was H, Dulak J. Heme oxygenase-1 in tumors: is it a false friend? Antioxid Redox Signal. 2007;9(12):2099-2117.

27. Doi K, Akaike T, Fujii S, et al. Induction of haem oxygenase-1 nitric oxide and ischaemia in experimental solid tumours and implications for tumour growth. Br J Cancer. 1999;80(12):1945-1954.

28. Miller SJ, Rangwala F, Williams J, et al. Large-scale molecular comparison of human schwann cells to malignant peripheral nerve sheath tumor cell lines and tissues. Cancer Res. 2006;66(5):2584-2591.

29. Johansson G, Mahller YY, Collins MH, et al. Effective in vivo targeting of the mammalian target of rapamycin pathway in malignant peripheral nerve sheath tumors. Mol Cancer Ther. 2008;7(5):1237-1245.

30. Liu XA, Liao K, Liu R, et al. Tau dephosphorylation potentiates apoptosis by mechanisms involving a failed dephosphorylation/activation of Bcl-2. J Alzheimers Dis. 2010;19(3):953-962.

31. Liu XA, Song J, Jiang Q, Wang Q, Tian Q, Wang JZ. Expression of the hyperphosphorylated tau attenuates ER stress-induced apoptosis with upregulation of unfolded protein response. Apoptosis. 2012; 17(10):1039-1049

32. Liu XA, Zhu LQ, Zhang Q, et al. Estradiol attenuates tau hyperphosphorylation induced by upregulation of protein kinase-A. Neurochem Res. 2008;33(9):1811-1820.

33. Abraham NG, Lavrovsky Y, Schwartzman ML, et al. Transfection of the human heme oxygenase gene into rabbit coronary microvessel endothelial cells: protective effect against heme and hemoglobin toxicity. Proc Natl Acad Sci U S A. 1995;92(15):6798-6802.

34. Leger DY, Liagre B, Beneytout JL. Role of MAPKs and NF-kappaB in diosgenin-induced megakaryocytic differentiation and subsequent apoptosis in HEL cells. Int J Oncol. 2006;28(1):201-207.

35. Yadav VR, Prasad S, Sung B, Kannappan R, Aggarwal BB. Targeting inflammatory pathways by triterpenoids for prevention and treatment of cancer. Toxins (Basel). 2010;2(10):2428-2466.

36. Johnson GL, Lapadat R. Mitogen-activated protein kinase pathways mediated by ERK, JNK, and p38 protein kinases. Science. 2002; 298(5600):1911-1912.

37. Ryter SW, Choi AM. Heme oxygenase-1: molecular mechanisms of gene expression in oxygen-related stress. Antioxid Redox Signal. 2002;4(4):625-632.

38. Bloom DA, Jaiswal AK. Phosphorylation of Nrf2 at Ser40 by protein kinase $\mathrm{C}$ in response to antioxidants leads to the release of Nrf2 from INrf2, but is not required for Nrf2 stabilization/accumulation in the nucleus and transcriptional activation of antioxidant response elementmediated NAD(P)H:quinone oxidoreductase-1 gene expression. J Biol Chem. 2003;278(45):44675-44682.

39. Qi D, Ouyang C, Wang Y, et al. HO-1 attenuates hippocampal neurons injury via the activation of BDNF-TrkB-PI3K/Akt signaling pathway in stroke. Brain Res. 2014;1577:69-76.

40. Nahirnyj A, Livne-Bar I, Guo X, Sivak JM. ROS detoxification and proinflammatory cytokines are linked by p38 MAPK signaling in a model of mature astrocyte activation. PLoS One. 2013;8(12):e83049. 
41. Cheng S, Du Y, Bing F, Zhang G. Synthesis of flaccidoside II, a bidesmosidic triterpene saponin isolated from Chinese folk medicine Di Wu. Carbohydr Res. 2008;343(3):462-469.

42. Merritt AJ, Potten CS, Watson AJ, Loh DY, Nakayama K, Hickman JA. Differential expression of bcl-2 in intestinal epithelia. Correlation with attenuation of apoptosis in colonic crypts and the incidence of colonic neoplasia. J Cell Sci. 1995;108(pt 6):2261-2271.

43. Huang F, Cao J, Liu Q, Zou Y, Li H, Yin T. MAPK/ERK signal pathway involved expression of COX-2 and VEGF by IL-1beta induced in human endometriosis stromal cells in vitro. Int J Clin Exp Pathol. 2013; 6(10):2129-2136.

44. Lin CC, Hsieh HL, Shih RH, Chi PL, Cheng SE, Yang CM. Upregulation of COX-2/PGE2 by endothelin-1 via MAPK-dependent NF-kappaB pathway in mouse brain microvascular endothelial cells. Cell Commun Signal. 2013;11(1):8.

45. Zhang W, Liu HT. MAPK signal pathways in the regulation of cell proliferation in mammalian cells. Cell Res. 2002;12(1):9-18.

46. Le WD, Xie WJ, Appel SH. Protective role of heme oxygenase-1 in oxidative stress-induced neuronal injury. J Neurosci Res. 1999;56(6): 652-658.

47. Takeda A, Perry G, Abraham NG, et al. Overexpression of heme oxygenase in neuronal cells, the possible interaction with Tau. J Biol Chem. 2000;275(8):5395-5399.
48. Chau LY. Heme oxygenase-1: emerging target of cancer therapy J Biomed Sci. 2015;22:22.

49. Zhao M, Guo H, Chen J, et al. 5-aminolevulinic acid combined with sodium ferrous citrate ameliorates $\mathrm{H} 2 \mathrm{O} 2$-induced cardiomyocyte hypertrophy via activation of the MAPK/Nrf2/HO-1 pathway. Am J Physiol Cell Physiol. 2015;308(8):C665-C672.

50. Kim JK, Jang HD. Nrf2-mediated HO-1 induction coupled with the ERK signaling pathway contributes to indirect antioxidant capacity of caffeic acid phenethyl ester in HepG2 cells. Int J Mol Sci. 2014;15(7): 12149-12165.

51. Xu XH, Li GL, Wang BA, et al. Diallyl trisufide protects against oxygen glucose deprivation-induced apoptosis by scavenging free radicals via the PI3K/Akt-mediated Nrf2/HO-1 signaling pathway in B35 neural cells. Brain Res. 2015;1614:38-50.

52. Lee SE, Jeong SI, Yang H, Park CS, Jin YH, Park YS. Fisetin induces Nrf2-mediated HO-1 expression through PKC-delta and p38 in human umbilical vein endothelial cells. J Cell Biochem. 2011;112(9): 2352-2360.

53. Gong $\mathrm{P}$, Cederbaum AI, Nieto N. Increased expression of cytochrome P450 2E1 induces heme oxygenase-1 through ERK MAPK pathway. J Biol Chem. 2003;278(32):29693-29700.
OncoTargets and Therapy

\section{Publish your work in this journal}

OncoTargets and Therapy is an international, peer-reviewed, open access journal focusing on the pathological basis of all cancers, potential targets for therapy and treatment protocols employed to improve the management of cancer patients. The journal also focuses on the impact of management programs and new therapeutic agents and protocols on

\section{Dovepress}

patient perspectives such as quality of life, adherence and satisfaction. The manuscript management system is completely online and includes a very quick and fair peer-review system, which is all easy to use. Visit http://www.dovepress.com/testimonials.php to read real quotes from published authors. 